\title{
AN ORGANIZING PRINCIPLE FOR A CLASS OF VOLUNTARY MOVEMENTS $^{1}$
}

\author{
NEVILLE HOGAN
}

\author{
Department of Mechanical Engineering, Massachusetts Institute of Technology, Cambridge, Massachusetts 02139
}

Received December 12, 1983; Revised May 29, 1984; Accepted May 30, 1984

\begin{abstract}
This paper presents a mathematical model which predicts both the major qualitative features and, within experimental error, the quantitative details of a class of perturbed and unperturbed large-amplitude, voluntary movements performed at intermediate speed by primates. A feature of the mathematical model is that a concise description of the behavioral organization of the movement has been formulated which is separate and distinct from the description of the dynamics of movement execution.

Based on observations of voluntary movements in primates, the organization has been described as though the goal were to make the smoothest movement possible under the circumstances, i.e., to minimize the accelerative transients. This has been formalized by using dynamic optimization theory to determine the movement which minimizes the rate of change of acceleration (jerk) of the limb.

Based on observations of muscle mechanics, the concept of a "virtual position" determined by the active states of the muscles is rigorously defined as one of the mechanical consequences of the neural commands to the muscles. This provides insight into the mechanies of perturbed and unperturbed movements and is a useful aid in the separation of the descriptions of movement organization and movement execution.
\end{abstract}

This paper presents a mathematical model of a class of voluntary arm movements observed in monkeys. The movements which have been considered are large-amplitude, singlejoint, elbow motions at intermediate speed. There are, of course, many possible mathematical models of any given phenomenon; the work presented here is an attempt to capture in a single description both the major global features of the way the movement is organized and the finer quantitative details of the way the movement is executed. Accordingly, dynamic optimization theory (Bryson and Ho, 1975) has been used. Briefly, the technique is to define a criterion function (see below) which models the goal or objective of the movement. The criterion function assigns a real number to every possible movement trajectory. A differential equation is also formulated which models the relation between system outputs (e.g., positions) and inputs (e.g., neural activities). The methods of variational calculus are then applied to find from the infinite set of possible trajectories the one which minimizes the criterion function subject to the dynamic constraints imposed by the differential equations and the conditions imposed at the boundaries (i.e., the start and finish of the movement). In this paper a criterion function is presented which adequately describes observed behavior and which may represent an organizing principle underlying voluntary movement.

${ }^{1}$ This research was supported by National Institute of Neurological Disease and Stroke Research Grant NS09343, National Institute of Arthritis, Metabolism, and Digestive Diseases Grant AM26710, National Aeronautics and Space Administration Grant NAG 2-126, and National Eye Institute Grant EY02621. I am indebted to Drs. Emilio Bizzi, Neri Accornero, William Chapple, and Tomaso Poggio, and Ms. Shiela Cotter for their many insightful comments on this work.
The mathematical model presented here provides an interpretation of single- and multi-joint movements performed by primates. The generalization of the work presented in this paper to multi-joint movement has recently been presented by Flash and Hogan (1982). The question of the relation of this mathematical model to current neurophysiological models such as "final position control" and in particular to the body of myoelectric and single cell recordings is dealt with under "Discussion" and in Bizzi et al. (1984).

\section{System Equations: The Virtual Trajectory}

The differential equations used to describe the system behavior are based on experimental observations of intact and deafferented monkeys making voluntary pointing movements of the forearm to a visually presented target. It should be pointed out that these are large movements performed at intermediate speed. The behavior to be modeled has been described in detail elsewhere (Bizzi et al., 1981a, b, 1982a, b, 1984). For present purposes a key observation culled from these data is that perturbations applied before and after a movement demonstrated that the limb was in a stable cquilibrium posture, even in the absence of afferent feedback.

In the theory presented in this paper the elbow joint is assumed to have a constant center of rotation and the forearm is treated as a rigid body. The entire group of flexor and extensor muscles generates a single resultant torque about the elbow joint. Although muscle force is a complicated function of many variables, the dominant mechanical behavior may be represented by considering only its dependence on the muscle length and its rate of change. Accordingly, the resultant torque may be assumed to depend only upon the active states of the muscles spanning the joint and upon the angular position and 
velocity of the joint. The resulting differential equation describing forearm motion is:

$$
I \ddot{\theta}=T(\theta, \dot{\theta},\{a\})
$$

where $\theta$ is elbow angular position, $\dot{\theta}$ is elbow angular velocity, $\ddot{\theta}$ is elbow angular acceleration, $\{a\}$ is the set of muscle active states, $I$ is forearm inertia, $T$ is resultant muscle torque, and parentheses denote functional dependence.

At equilibrium the velocity and acceleration of the limb are both zero, and thus, the resultant torque about the elbow joint in the absence of external torques is also zero:

$$
T(\theta,\{a\}) \mid \begin{aligned}
& \dot{\theta}=0 \\
& \ddot{\theta}=0
\end{aligned}=0
$$

'The observation that an equilibrium posture of the limb may be maintained even for a brief period (e.g., $1 \mathrm{sec})$ in the absence of feedback (Kelso, 1977; Polit and Bizzi, 1978, 1979; Bizzi and Polit, 1979; Kelso and Holt, 1980; Schmidt and McGown, 1980; Bizzi et al., 1981a, b, 1982a, b, 1984) means that this equation may be solved explicitly to express the equilibrium position of the limb as a function of the set of muscle active states. Note that the duration of the period over which the equilibrium posture is maintained does not affect the solvability of the equation

$$
\theta o=\theta o(\{a\})
$$

where $\theta 0$ is equilibrium position.

In other words, a change in $\{a\}$ requires a corresponding change in $\theta$ to maintain $T=0$. Now assume that the functional form of the relation between joint torque and forearm motion (equation 1) remains the same during movement (although its parameters may change). As a result, the set of muscle active states may always be interpreted as defining a virtual equilibrium position for the limb. When the muscle activity varies, a time-history of virtual positions is generated which is termed the virtual trajectory.

Muscle activity may of course determine more than just an equilibrium position for the limb. For example, the net mechanical impedance ${ }^{2}$ about the joint may be modulated by the coactivation of antagonist muscles (Hogan, 1979, 1980a, b, 1984; Lanman 1980). The concept of a virtual position is merely a representation of one of the mechanical consequences of a given set of muscle activities. If the limb is in motion the virtual position defined by the set of muscle active states may differ from the actual position of the forearm. Indeed, it may even lie outside of the range of reachable positions for the limb. The virtual position may conveniently be thought of as that position toward which the instantaneous set of muscle activities is driving the limb at any point in time.

It is important to note that the concept of a virtual position or trajectory does not require any assumptions of linearity of the relation between muscle force and length or velocity of shortening. However, in part of the work presented below, a numerical description of the dependence of resultant torque on angular position and velocity is required. In the interest of simplicity, in that part of the paper it will be assumed that the position and velocity dependencies are uncoupled and linear. These assumptions are made explicit as follows:

$$
T(\theta, \dot{\theta},\{a\})=T(\{a\})-K \theta-B \dot{\theta}
$$

where $K$ is angular stiffness and $B$ is angular viscosity.

An expression for the virtual trajectory is found by applying equation 2.

$$
\begin{aligned}
& T(\{a\})=K \theta \\
& \theta o(\{a\}=T(\{a\}) / K
\end{aligned}
$$

\footnotetext{
${ }^{2}$ Mechanical impedance refers to the dynamic equation relating resulting forces and torques to imposed motion and includes stiffness, viscosity, etc.
}

Rewriting in terms of the virtual trajectory we obtain a simple second-order equation as the model of the forearm dynamics:

$$
I \ddot{\theta}+B \dot{\theta}+K \theta=K \theta o(\{a\})
$$

A further simplifying assumption is to equate the active state of the muscle to its input alpha motoneuron activity, in effect neglecting the dynamics of the physiological processes which produce the mechanical output of the muscle in response to the input neural activity. This assumption preserves the secondorder form of the equations. In the following it will be shown that this simplified, linear, second-order model can adequately describe the observed behavior.

\section{The Criterion Function: Minimum-Jerk Motion}

Equation 7 models the output motion of the forearm in response to a specified alpha motoneuron input. An equally important part of a model of the complete system is the procedure for selecting appropriate inputs. In the following the time-varying inputs to the differential equations are determined by using dynamic optimization techniques to minimize a criterion function. Minimization of the criterion function is a mathematical model of the behavioral organization of the movement.

The key element in this part of the system model is the criterion function and, as before, the choice was based on experimental observations. The smoothness and gracefulness of the actual motions performed by intact ${ }^{3}$ primates is suggestive and the organizing principle assumed in the following analysis may be stated as follows: "Generate the smoothest motion which will bring the limb from equilibrium at the starting position to equilibrium at the target position in a given time."

It is assumed that maximizing the smoothness may be modeled by minimizing the mean-square jerk. Jerk is defined mathematically as the third time derivative of position, or the rate of change of acceleration. The criterion function to be minimized is thus:

$$
C=\int_{o}^{d} \gamma^{2} / 2 d t
$$

where $C$ is the criterion function, $t$ is time, $d$ is duration of movement, and $\gamma$ denotes jerk.

As discussed later, additional terms could be included in the criterion function, but they are not essential. Hence, for clarity, only the mean-square jerk will be considered in this paper.

\section{Dynamic Optimization}

Dynamic optimization refers to the use of the calculus of variations to minimize a functional subject to a set of constraining dynamic equations and algebraic equalities or inequalities. A comprehensive introduction to the topic is to be found in Bryson and Ho (1975) and Pontryagin et al. (1962). In this paper, a trajectory is to be found which brings the value of the criterion function (equation 8) to a minimum subject to the constraints imposed by the dynamics of the system (equation 7) and its physical limitations such as the maximum torque the muscles may exert or the maximum speed of the limb. The method of Pontryagin et al. (1962) is applied in Appendix I to derive necessary conditions for the existence of a minimum. The form of the minimum-jerk movement trajectory is a fifthorder polynomial in time:

$$
\theta(t)=b_{0}+b_{1} t+b_{2} t^{2}+b_{3} t^{3}+b_{4} t^{4}+b_{5} t^{5}
$$

where $b_{0} \ldots b_{5}$ are constants.

\footnotetext{
${ }^{3}$ Single-joint forearm movements in deafferented monkeys show acceleration profiles similar to those observed in the movements of intact animals (Bizzi et al., 1984). They are smooth in the sense that the accelerative transients are kept to a minimum. This is precisely the meaning of "smooth" which is quantified by equation 8 .
} 
The details of a specific movement depend on its boundary conditions. A sufficient set of boundary conditions consists of the position, velocity, and acceleration at the start of the movement (time $=0$ ) and at the finish (time $=d$ ). The movements to be modeled start and finish at rest; thus the chosen boundary conditions ${ }^{4}$ for this point-to-point movement are as follows:

$$
\begin{array}{lll}
\theta(0)=p s ; & \dot{\theta}(0)=0 ; & \ddot{\theta}(o)=0 \\
\theta(d)=p f ; & \dot{\theta}(d)=0 ; & \ddot{\theta}(d)=0
\end{array}
$$

where $p s$ is position of start and $p f$ is position of finish.

Solving for the constants $b 0 \ldots b 5$ yields the following equation for the movement:

$$
\theta(t)=p s+a\left\{10(t / d)^{3}-15(t / d)^{4}+6(t / d)^{5}\right\} 0 \leq t \leq d
$$

where $a$ is movement amplitude, and $a=p f-p s$.

Equation 11 shows that the shape of the predicted movement trajectory does not change with amplitude or duration of the movement. Changes in movement amplitude or duration merely serve to change the scale of the position and time axes, respectively. The position, velocity, and acceleration profiles for a $60^{\circ}$ movement lasting $700 \mathrm{msec}$ are shown in Figure 1. The following characteristics of the movement may be derived:

$$
\begin{aligned}
& \text { Maximum velocity }=1.88 \mathrm{a} / \mathrm{d} \\
& \text { Maximum acceleration }=5.77 \mathrm{a} / \mathrm{d}^{2}
\end{aligned}
$$

The observations presented by Bizzi et al. (1982a, b, 1984) of monkeys performing undisturbed pointing movements show a measured mean value of peak absolute acceleration for a $60^{\circ}$ movement of $1130^{\circ} / \mathrm{sec}^{2}$. The variability was such that 1 SD about the mean covered a range from 1450 to $810^{\circ} / \mathrm{sec}^{2}$. A minimum-jerk motion with the same amplitude and peak absolute acceleration has a duration of $554 \mathrm{msec}$ with 654 and $489 \mathrm{msec}$ representing the corresponding range of deviations. The measured distribution of movement durations was skewed toward shorter times with a mean value of $692 \mathrm{msec}$ and a standard deviation of $676 \mathrm{msec}$.

It can be seen from equation 13 that if the movement duration remains constant, the peak absolute acceleration will scale with movement amplitude. A $20^{\circ}$ minimum-jerk movement of 554 msec duration will have a peak acceleration of $376^{\circ} / \mathrm{sec}^{2}$. Experimental measurements of $20^{\circ}$ movements show a mean peak absolute acceleration of $397^{\circ} / \mathrm{sec}^{2}$ (Bizzi et al., 1984). Within the limits of experimental accuracy the minimum-jerk movement profile yields good qualitative and quantitative agreement with observed undisturbed movement profiles.

The experimental observations make it clear that the animal is not operating anywhere near the limits of neuromuscular performance, and this can be used to gain insight into the mathematical results. The chosen criterion function (equation 8) which models the organization of the movement depends only on kinematic variables related to the trajectory of movement. If a movement lies within the performance envelope of the neuromuscular system, the problem of minimizing equation 8 is effectively unconstrained and the classical techniques of variational calculus may be applied to derive the optimal movement trajectory directly (see Appendix II).

Consequently, the result presented in equation 11 represents a planned movement the coordination or organization of which is completely independent of the physical system generating the motion. This is in keeping with the idea originally proposed by Bernstein (for a recent re-examination of Bernstein's work see Whiting, 1984) and supported by recent experimental work (Morasso, 1981) that movements are coordinated in terms of the motion of the hand in extracorporeal space. Furthermore, because no model of the neuromuscular system is required and assumptions of linearity or nonlinearity are irrelevant, this

${ }^{4}$ Other boundary conditions are of course possible. Nelson (1983) used a different set to describe repetitive, reciprocating movements.
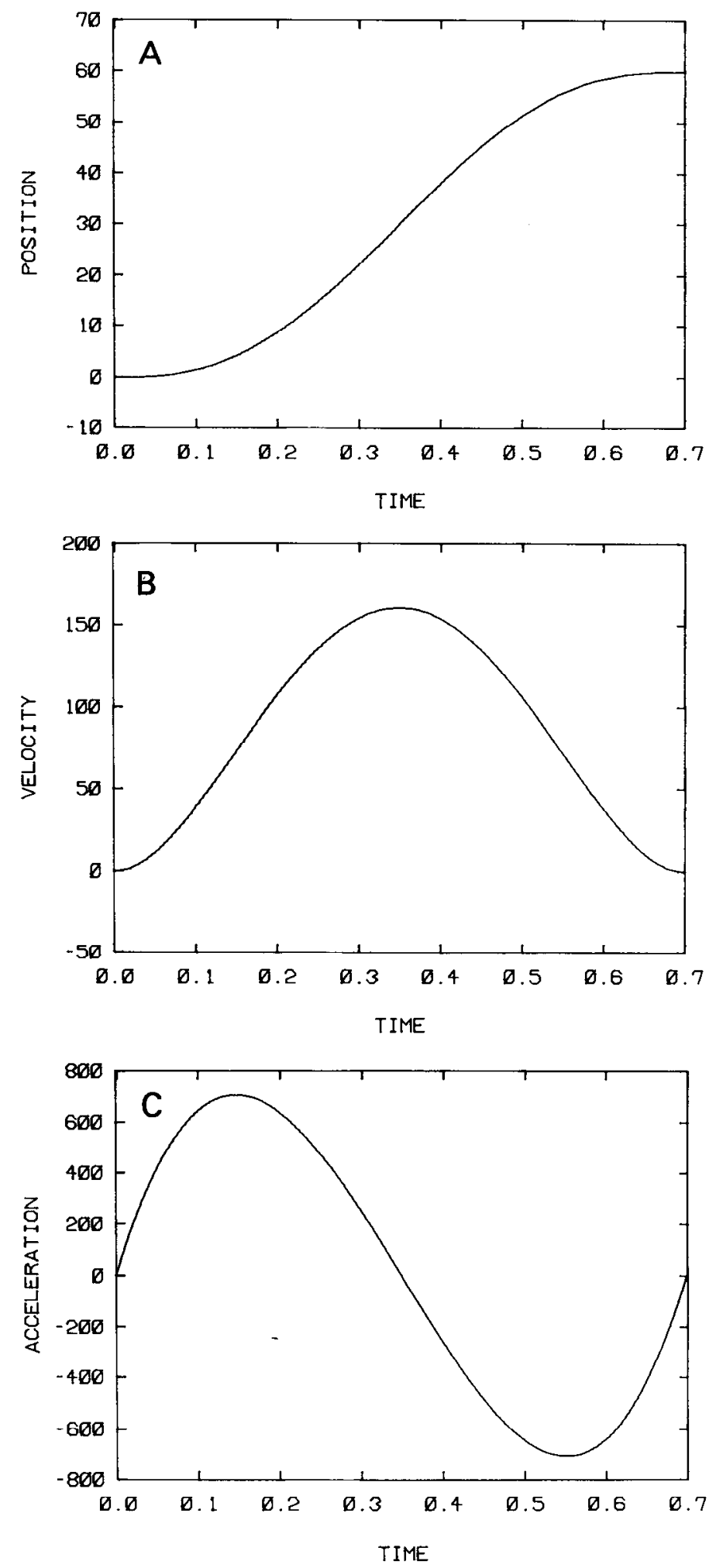

Figure 1 . The position $(A)$, velocity $(B)$, and acceleration $(C)$ profiles for a $60^{\circ}$ minimum-jerk motion of $700 \mathrm{msec}$ duration between two equilibrium posilions. Note the bell-shaped velocity profile characteristic of voluntary movements in primates. Horizontal axes represent time in seconds; vertical axes represent $(A)$ degrees, $(B)$ degrees per second, and $(C)$ degrees per second ${ }^{2}$.

model of the organization of movement would be expected to have considerable generality-provided the resulting movement lies within the performance envelope of the system. An extension of this model to multi-joint movements has been presented by Flash and Hogan (1982). 


\section{Digital Simulation}

In contrast to the actual movement profile, the prediction of the virtual trajectory depends heavily on the assumed model of the neuromuscular system. To obtain numerical predictions, the linear model of equation 7 was used. As this model has been kept as simple as possible, only three parameter values are needed: the inertial, viscous, and elastic coefficients for the forearm. The inertia was measured directly by applying small, brief pulses of torque to the forearm while it was at rest and measuring the resulting initial acceleration. The value $I=0.014$ kilogram-meter ${ }^{2}$ was obtained.

The elastic coefficient was estimated as follows. Experiments were performed in which the forearm was held at rest in its initial position and released at various times following the onset of evoked agonist myoelectric activity (Bizzi et al., 1984). The acceleration of the limb just at the moment of release was measured. The torque causing this acceleration was obtained by multiplying by the inertia. As the velocity of the limb was zero at the moment of release, any viscous torque was zero, and according to the model the accelerating torque was solely due to the elastic torque generated by the difference between the virtual position and the actual position. In addition, according to the model, at (or after) the end of a movement the virtual position coincides with the larget position. Thus, when the limb was held in the start position up to (or beyond) the duration of a normal unperturbed movement, the angular displacement of target from start equalled the difference between the virtual and actual positions. Dividing the accelerating torque by the angular displacement of target from start yielded an estimate of the stiffness.

The experimental results (Bizzi et al., 1984) showed that, when the limb was held for $700 \mathrm{msec}$ (equal to or exceeding the duration of most normal unperturbed movements), the accel- eration at the moment of release was about $40 \mathrm{radian} / \mathrm{sec}^{2}$. This requires an initial accelerating torque of 0.56 newton-meter and yields an estimated stiffness of 0.64 newton-meter/radian. Accordingly, in the linear, second-order equation describing the forearm dynamics an undamped natural frequency of 6.77 radian/sec $(1.08 \mathrm{~Hz})$ was used.

The application of torque pulses to the limb when it was at rest showed that the system was underdamped (damping ratio between 0.1 and 1.0 approximately), but it did not prove practical to measure the viscous coefficient directly. Instead, for a series of values of the damping ratio digital computations of the time course of the virtual trajectory were performed and compared with the results of the hold-and-release experiment.

Figure 2 shows a plot of the experimental results superimposed upon plots of the predicled virlual trajectory. To compare the theoretical prediction with the experimental observations, the virtual trajectory (an angular displacement) has been multiplied by the estimated stiffness to yield a torque and divided by the inertia to yield an acceleration. It can be seen that the simple model developed above provides an adequate fit to the experimental data and that the match between prediction and experiment is not unduly sensitive to the value chosen for the damping ratio.

Using a damping ratio of 0.4 , digital simulations of the actual forearm movement were performed. A simulated unperturbed movement and the virtual trajectory required to generate it are shown in Figure $3 A$. Note that because of the spring-like behavior of the muscles the virtual position must initially precede the actual position of the forearm in order to generate the torques necessary to produce the desired movement.

Experiments were performed in which the limb was driven by servo action into the target position and released from there at the onset of evoked myoelectric activity (Bizzi et al., 1984). To simulate the effect of this perturbation a computation was

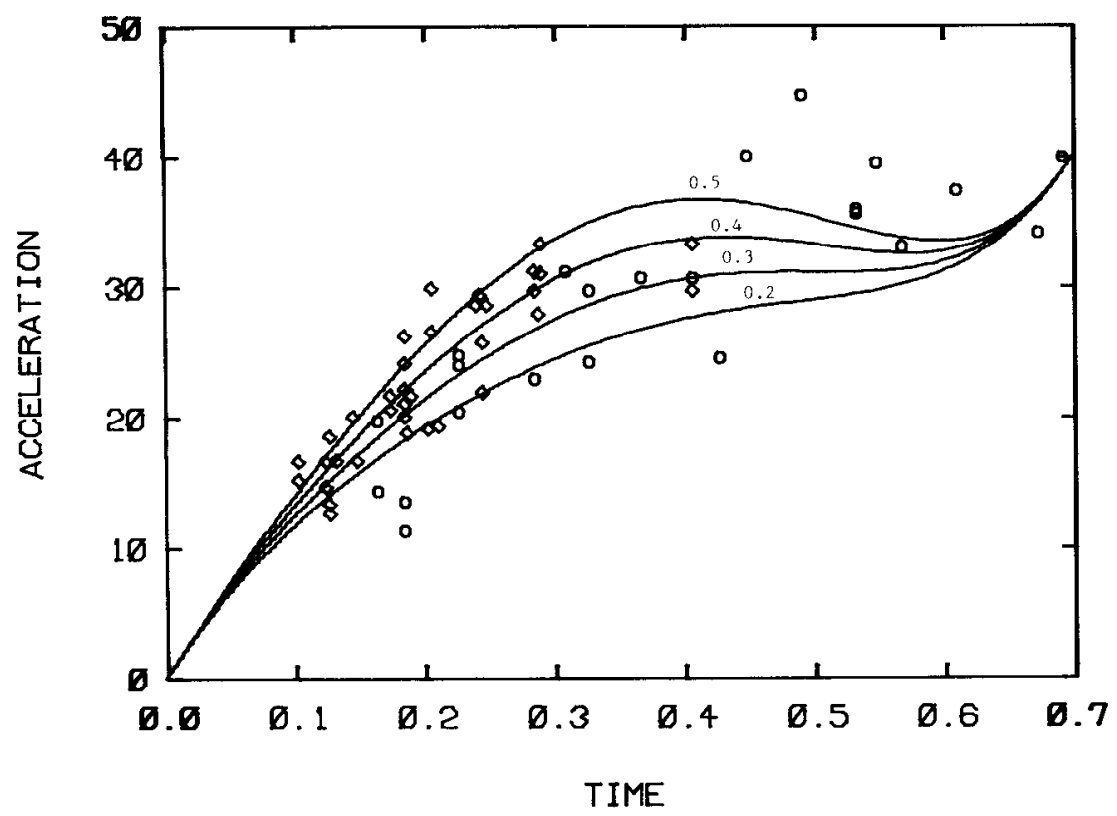

Figure 2. The smooth curves are the simulated virtual trajectories required to generate a movement lasting $700 \mathrm{msec}$ when the forearm dynamics are given by equation 7 with an undamped natural frequency of $6.77 \mathrm{radian} / \mathrm{sec}$ and damping ratios of $0.2,0.3,0.4$ and 0.5 , respectively. The points are experimental data reported by Bizzi et al. (1984) on the initial acceleration of a monkey's forearm upon release after being held for a period of time following the onset of voluntary muscle activity (diamonds represent intact animals, and circles represent deafferented animals). For comparison with the experimental data, the virtual trajectories have been multiplied by the stiffness and divided by the inertia of the forearm to yield an acceleration. The horizontal axis represents time in seconds; the vertical axis represents radian per second ${ }^{2}$. 


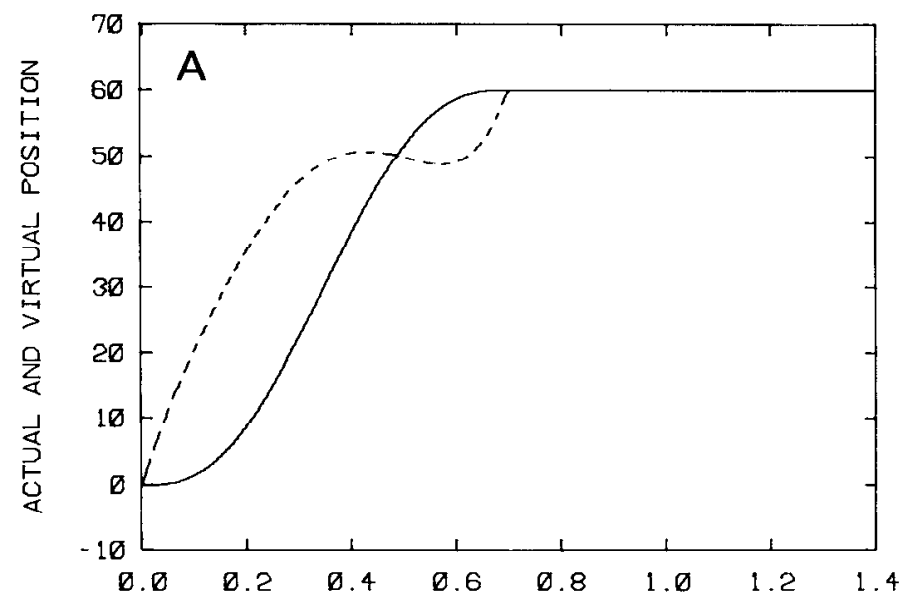

TIME

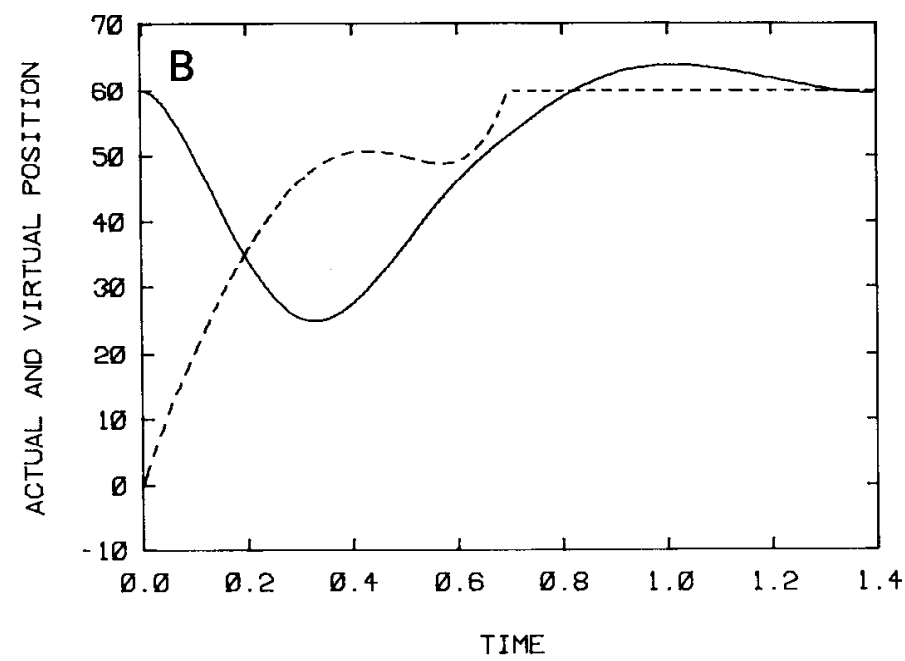

Figure 3. A digital simulation of the actual (solid lines) and virtual (dashed lines) trajectories for an undisturbed $60^{\circ}$ motion lasting 700 msec is shown in $A$. The same virtual trajectory and the actual trajectory which results when the limb is released from rest at the target position at time zero are shown in $B$. Note that, although the input to the system (the virtual trajectory) has remained unchanged, the limb first heads back toward the initial position before reversing direction and proceeding to the target position. Horizontal axes represent time in seconds; vertical axes represent degrees.

performed using the same parameters and the same virtual trajectory, but with different initial conditions such that the forearm started from rest at the target position. Figure $3 B$ shows the result: as observed in the experimental animals, the limb first moved backwards toward the starting position before changing direction and proceeding to the target.

Note that the model of equation 7 does not explicitly describe any modification of the alpha motoneuron activities due to sensory feedback. Any contribution of muscle spindle or tendon organ feedback to the apparent stiffness or viscosity of the forearm is included with the inherent stiffness and viscosity of the musculature and ligature in the model parameters $K$ and $B$. Consequently, the model predicts that the same alpha moloneuron activity which generated the virtual trajectory and actual movement of Figure $4 A$ will also generate the movement of Figure $4 B$ when only the initial conditions are changed. Because the muscles are defining an equilibrium position (virtual position) for the limb, the initial motion of the limb is to move toward the instantaneous value of the virtual position, even though this may imply an extensor motion in the presence of predominant flexor activity (or vice versa).

The important point here is that the extensor motion in the presence of predominant flexor activity (or vice versa) implies that the virtual position undergoes a gradual transition between start and final equilibrium positions. Most of the transition occupies about half of the movement duration. The simulated virtual trajectory reaches a local maximum at $430 \mathrm{msec}$ and stays near the final position (less than $20 \%$ of the movement amplitude away from it) thereafter.

\section{Discussion}

The observation in both the intact and deafferented animals of extensor motion during predominant flexor activity (or vice versa) is a compelling reminder of the fact that muscle may not be viewed simply as a pure force generator. The concept of a "virtual position" defined by the relevant muscles is a concise and effective way of including the length dependence in a model of muscle. The active states of the relevant muscles may always be interpreted by specifying a position command (the virtual position). Torque is generated by the apparent elasticity of the musculature in response to the difference between the actual position and the virtual position.

The question of what muscle variables are commanded by the central nervous system in limb movements has been discussed at length in the literature (Stein, 1982). To compare and contrast the interpretation of neural commands underlying the mathematical model proposed in this paper with these alternative neurophysiological theories, note that, provided the relation between torque and angle can be inverted, it would be equally correct to interpret the active states as specifying a "virtual torque" with the limb position being specified by the compliance (the inverse of stiffness) of the limb in response to the difference between actual torque and virtual torque. If the torque-angle relation is invertible, it is meaningless to ask whether the central nervous system commands position or torque (two of the prominent alternatives which have been proposed); alpha motoneuron activity may equally well be interpreted as a position command (the virtual trajectory) or as a torque command. In the foregoing, the command was interpreted as a position for reasons of clarity as this permits direct comparison of the command with the actual position, and because it is theoretically more general-no assumption of an invertible torque-angle relation is required.

The numerical simulations presented in this paper match the observations presented by Bizzi et al. (1981a, b, 1982a, b, 1984), both qualitatively and (within experimental error) quantitatively. However, the model presented here is not exclusive; other models could be formulated to match the experimental data. For example, based on the experimental data shown in Figure 2, one might postulate a virtual trajectory which was a simple linear ramp from the start to the target position occupying about half of the movement duration. If this virtual trajectory is applied as input to the model of equation 7 using the parameters determined previously (undamped natural frequency of $6.77 \mathrm{radian} / \mathrm{sec}$ and damping ratio of 0.4 ), the resulting simulated motion exhibits a pronounced overshoot. The overshoot can be reduced by increasing the damping ratio, and because of the uncertainty in the value of the viscous coefficient, this change of parameter can be justified. Figure 4 shows a digital simulation of the response of the model to an input ramp lasting $400 \mathrm{mscc}$ using an undamped natural frequency of $6.77 \mathrm{radian} / \mathrm{sec}$ and a damping ratio of 0.7 as parameter values. As can be seen, there are some quantitative differences in the predictions - the simulated movement exhibits some overshoot and the undisturbed velocity and acceleration profiles are asymmetric-but the major qualitative features of the 

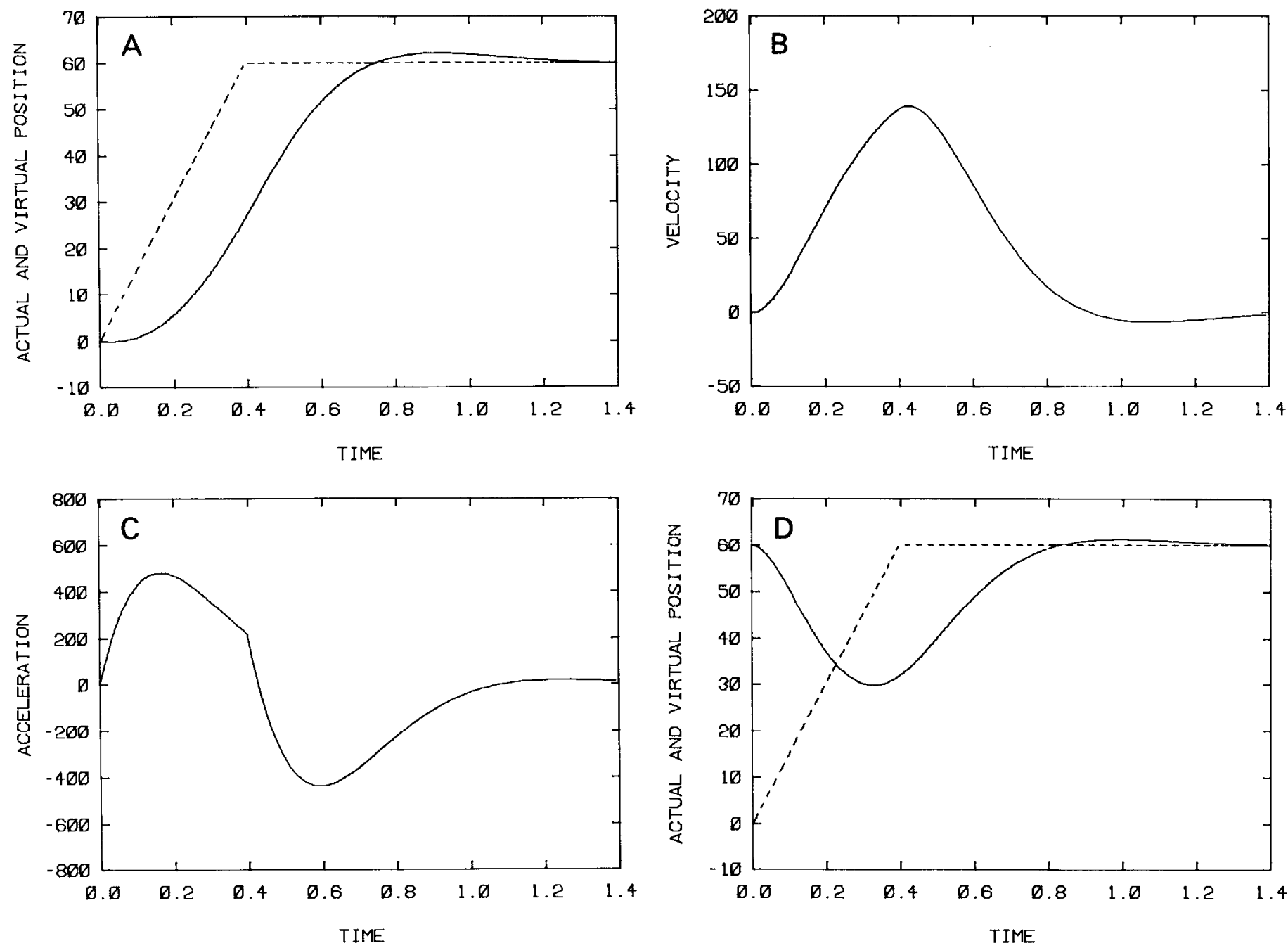

Figure 4. A digital simulation of the position $(A)$, velocity $(B)$, and acceleration $(C)$ profiles which result when the virtual position $($ dashed line) is a linear ramp from start to target positions lasting $400 \mathrm{msec}$. The dynamic parameters used are an undamped natural frequency of 6.77 radian/sec and a damping ratio of 0.7 . The corresponding position profile which results when the limb is released from rest at the target position is shown in $D$. Horizontal axes represent time in seconds; vertical axes represent: $(A)$ degrees, $(B)$ degrees per second, $(C)$ degrees per second ${ }^{2}$ and $(D)$ degrees.

movement, such as the reversal following the simulated perturbation, are produced. However, because it embodies a description of the organization of the movement, the minimum-jerk model has superior predictive capabilities. For example, if the planner were to specify a faster movement, the virtual trajectory would overshoot the target position (Fig. 5). As the virtual position is a function of the muscle active state, the difference between agonist and antagonist muscle activity would have a comparable time history, and this is not unlike the characteristic "pulse-step" patterns of myoelectric activity seen during rapid movements (Ghez and Vicario, 1978a, b; Ghez and Martin, 1982). The mathematical description also shows that one alternative strategy for generating faster movements would be to keep the form of the virtual trajectory unchanged, but scale the stiffness, damping, and duration such that the undamped natural frequency of the limb dynamics increases inversely proportional to the decrease in duration while the damping ratio remains constant (see Fig. 5). This may be experimentally testable and thus the mathematic analysis presented above may suggest some new avenues of investigation. Finally, the mathematical model presented here has considerable generality. It may be applied directly to other movements and more complex and physiologically realistic situations; the identical theoretical framework-minimization of mean-square jerk-has been successfully used to predict the detailed position and velocity profiles of multi-joint movements (Flash and Hogan, 1982; Flash, 1983).

The model of the neuromuscular system used in this paper neglected the dynamics of the excitation-contraction coupling. It might be argued that the observed gradual transition of the virtual position from the start to the target is not the execution of a motor plan, but merely a consequence of the sluggish response of muscle to an abrupt change in its neural input. This possibility cannot be ruled out, but it is difficult to reconcile with the known properties of muscle. An extremely conservative estimate of the response time of monkey biceps and triceps can be made by using data obtained from human biceps and triceps. The twitch contraction times of these muscles were reported by Buchthal and Schmalbruch (1970) to have mean values of 52 and $45 \mathrm{msec}$, respectively. As the net muscle force is the sum of a large number of twitch contractions produced by the input alpha motoneuron impulses, the twitch contraction can be regarded as the impulse response of a linear model of the muscle. A linear model with three identical poles at $40 \mathrm{radian} / \mathrm{sec}$ produces an impulse response (shown in Fig. $6 A$ ) with a time-to-peak contraction of $50 \mathrm{msec}$. The response 

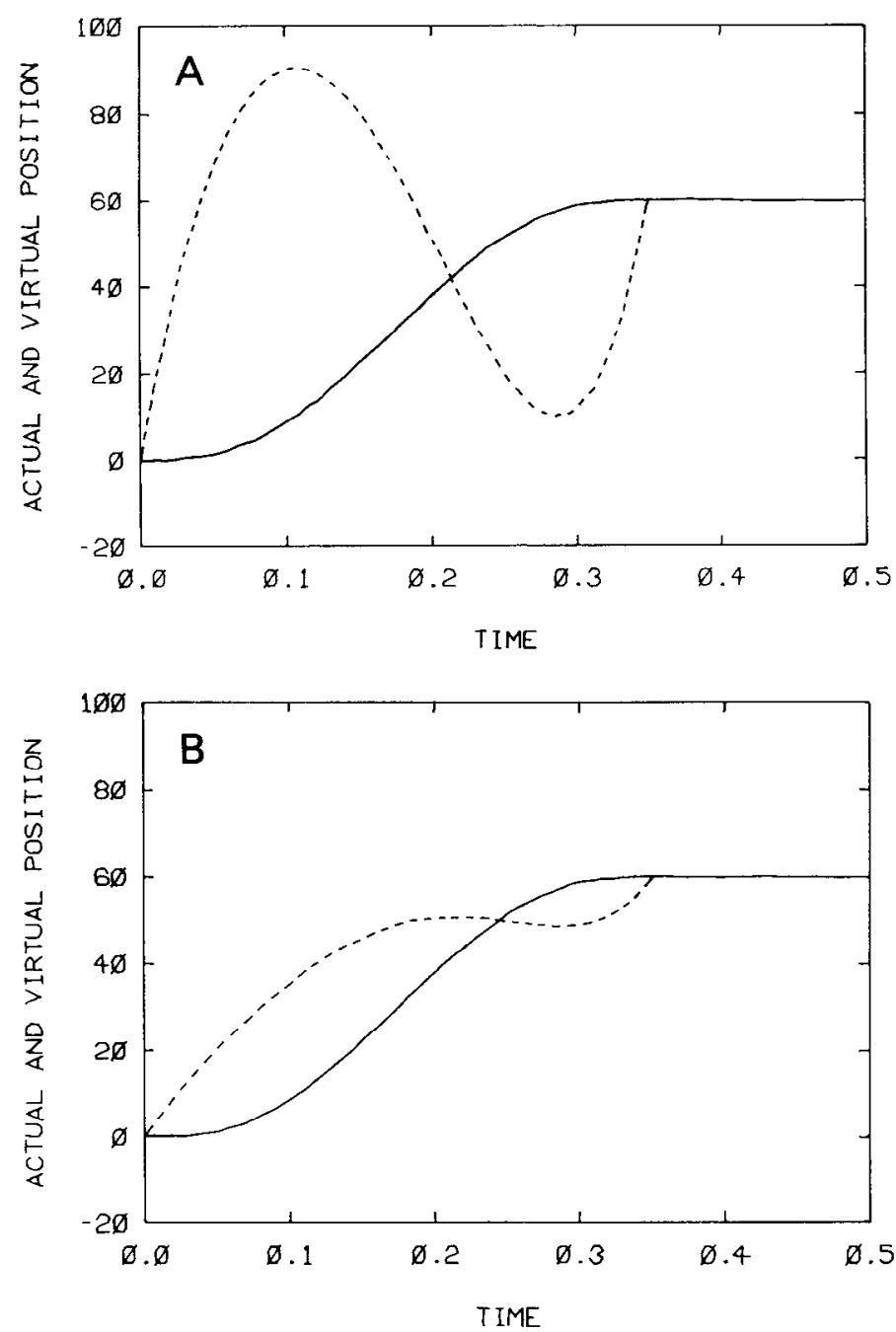

Figure 5. Two alternative strategies for controlling faster movements are simulated. One strategy is to modify the input. The virtual trajectory required to produce a minimum-jerk movement lasting $350 \mathrm{msec}$ when the limb dynamic parameters are unchanged (undamped natural frequency $6.77 \mathrm{radian} / \mathrm{sec}$, damping ratio 0.4 ) is shown in $A$. Note the initial overshoot or "pulse" in the virtual trajectory which is followed by a sustained "step." Another strategy is to modify the system dynamic parameters, leaving the shape of the virtual trajectory unchanged and simply scaling it with time. To produce a minimum-jerk movement lasting $350 \mathrm{msec}$ requires twice the undamped natural frequency (13.54 $\mathrm{radian} / \mathrm{sec}$ ) and the same damping ratio; i.e., a stiffness of 2.24 newtonmeters/radian and a viscosity of 0.142 newton-meter-second/radian. The corresponding virtual and actual trajectories are shown in $B$. Solid lines are simulated actual trajectories; dashed lines are virtual trajectories. Horizontal axes represent time in seconds; vertical axes represent degrees.

of this model of the excitation-contraction dynamics to a step change in neural input produces a "virtual trajectory" which rises to within a few percent of its final value within $200 \mathrm{msec}-$ considerably faster than the experimental data of Figure 2 indicate (see Fig. 6B).

Unfortunately, this simulation depends upon the order of the linear model assumed for the muscle contraction dynamics. A detailed in vivo experimental and theoretical study of the dynamics of human biceps and triceps was reported by Zahalak and Heyman (1979). They showed that a two-pole model with equal time constants of $50 \mathrm{msec}$ gave a good representation of their experimental data. The shape of the impulse response of this two-pole linear model of muscle contraction dynamics differs substantially from the shape of a typical twitch contraction-it exhibits a discontinuity in its initial rate of change (see Fig. 6C)-but for comparison purposes, this model was used to generate a virtual trajectory. The result is shown in Figure $6 \mathrm{D}$ : again the simulated virtual trajectory rises more rapidly than the experimental data of Figure 2, but the deviation from the experimental measurements is reduced. If the average time-to-peak twitch contraction of the motor units in monkey arm muscles were longer than $50 \mathrm{msec}$, this would further erode the difference between the simulations and the experimental data. Bearing in mind that the muscles of a monkey probably respond more rapidly than those of a human, the simulation results of Figures 6 show that it would be difficult to attribute the experimental observations to the contraction dynamics alone; but, without a reliable model of muscle, no definitive statement can be made. Given these considerations, it is clear that any comparison of this model with the data from single-cell recordings is premature at this stage.

Dynamic optimization is an essential part of the mathematical modeling presented in this paper. It permits global aspects of the movement to be factored into the analysis through the criterion function. It brings about a dramatic reduction in the dimensionality of the problem of choosing inputs for the differential equations. Of the infinite set of possible commands which would bring the system from start to target in the given time, only one minimizes the criterion function (provided the problem is non-singular). It provides a numerically testable model of the planning and organization of a voluntary movement which is complementary to the model of the dynamics of the neuromuscular system.

The heart of the dynamic optimization is the criterion function. It is a model of the organizational objective of the movement. Like all models, it is of necessity incomplete and could be refined and improved. The criterion function used in this analysis, that of minimizing the integral of the square of the jerk throughout the duration of the movement, was chosen as the simplest criterion function which would yield the observed motion profile. Other criterion functions have been investigated. Minimizing the mean-square snap (the fourth time derivative of position) has been shown to produce adequate predictions of experimentally observed movements, whereas minimizing mean-square acceleration does not (Flash, 1983). At the cost of increasing the complexity of the criterion function (and hence the analysis), the agreement between the model predictions and the experimental observations may be improved. The predicted motion profile has symmetrical acceleration and deceleration phases. However, it has been observed (Lanman, 1980; Morasso, 1981; Abend et al., 1982) that the acceleration phase of a point-to-point movement is occasionally somewhat shorter than the deceleration phase. This could readily be accommodated if the criterion function were augmented to include an additional term penalizing the square of the deviation of the actual position from the target position. This term would cause the controller to use higher levels of jerk and acceleration in the early portion of the movement so as to bring the error between actual and target positions down to a low value and use lower accelerations and jerks in the latter portion of the movement to offset this. The result is an asymmetric motion profile. However, an additional term in the criterion function would compromise the clarity and simplicity of the analysis, and as the observed asymmetry is typically small, including it was deferred for further research.

It is tempting to adduce physical or physiological reasons for minimizing jerk during a motion. For example, one might speculate that this strategy evolved to minimize "wear and tear" on the neuromuscular system. However, this is difficult to reconcile with the fact that the movements under consideration are at moderate speed and do not subject the animal to 

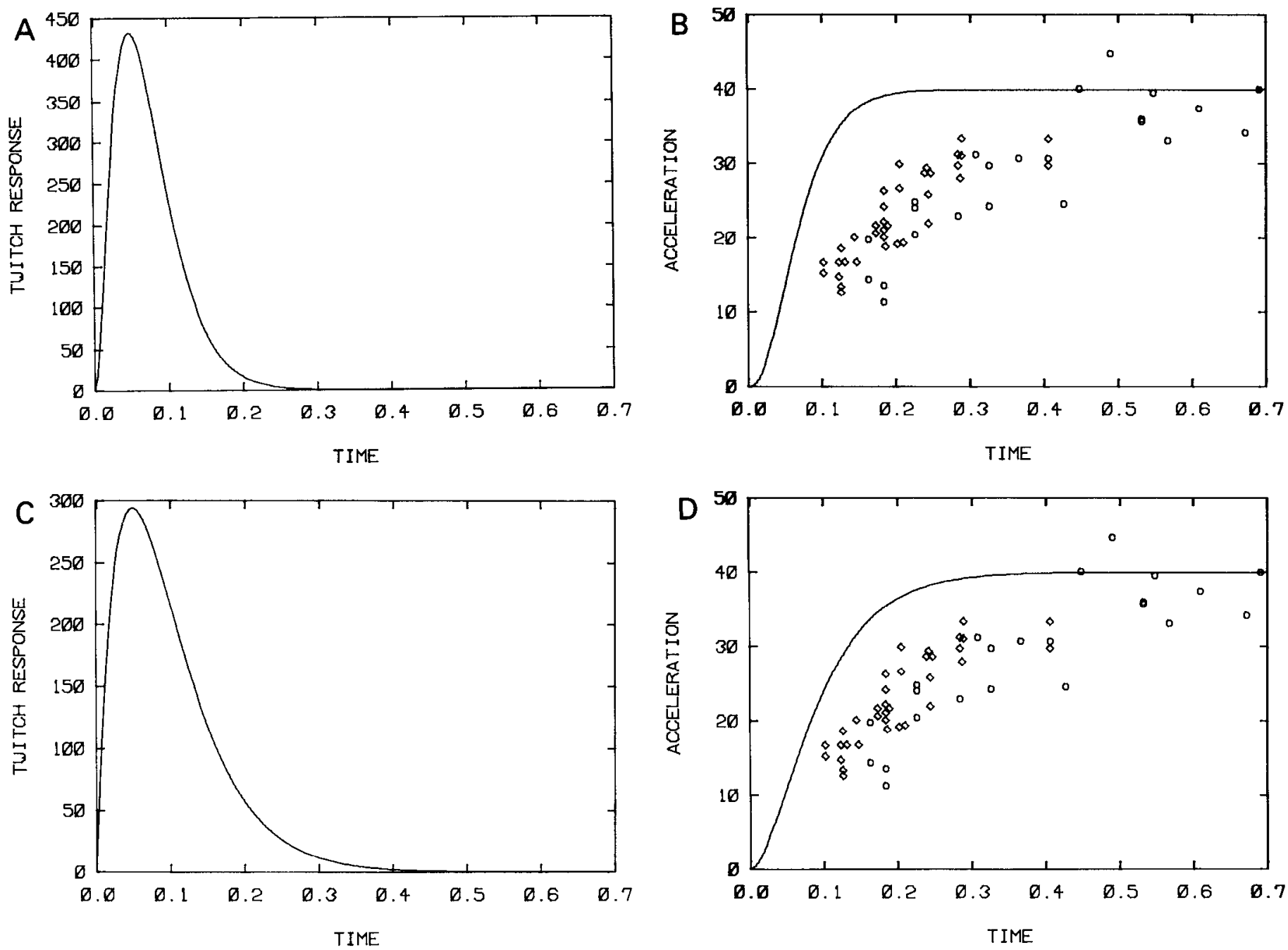

Figure 6. The twitch response of a three-pole linear model of the excitation-contraction dynamics of muscle is shown in $A$. The twitch response is simulated by applying an impulse input to the model. The three poles of the model are at $40 \mathrm{radian} / \mathrm{sec}$ resulting in a time-to-peak contraction of $50 \mathrm{msec}$. The virtual trajectory generated by this model in response to a step change in its input is shown in $B$, scaled as in Figure 2 for comparison with the experimental data of Figure 2. The twitch response of a two-pole linear model of the excitation-contraction dynamics of muscle is shown in $C$. The two poles of the model are at 20 radian sec resulting in a simulated twitch response time-to-peak contraction of 50 msec. The virtual trajectory generated by this model in response to a step change in its input is shown in $D$, scaled as in Figure 2 for comparison with the experimental data of Figure 2. Horizontal axes represent time in seconds; vertical axes represent: $(A)$ and $(C), 1 /$ sec; $(B)$ and $(D)$, radian/second ${ }^{2}$.

undue stress. Instead, it seems that minimizing jerk may simplify the control of the system. Reducing the magnitude of the higher derivatives of the motion implies a reduction in the amount of information required to specify, store, or predict the trajectory.

Whatever its physiological underpinnings, the real strength of the minimum-jerk criterion function, or indeed any other criterion function, is its use as an organizing principle. The use of variational principles is common in physics and engineering. They are not presented as the cause of the behavior they describe but rather as a distillation of its essence. The minimization of jerk provides a concise description of a wide variety of movements. Motion profiles similar to those derived in this paper can be seen in the data for point-to-point movements ranging from saccadic eye movements to movements of the entire upper arm (Clark and Stark, 1975; Viviani and Terzuolo, 1980; Morasso, 1981). Nelson (1983) has shown that an accurate prediction of repetitive arm movements during violin bowing and jaw movements during speech may be obtained from a minimum-jerk formulation by changing the boundary condi- tions so that acceleration is non-zero at the beginning and end of the movement. The minimum-jerk criterion function is also capable of predicting the major features of more complex voluntary behavior such as the trajectory of the hand in a planar motion around a visually presented obstacle (Abend et al., 1982; Flash and Hogan, 1982; Flash, 1983). Consequently, the mathcmatical analysis presented in this paper may be a first step toward a unified and general description of the organization of voluntary movements.

\section{Appendix I}

The objective function

$$
C=\int_{0}^{d} \gamma^{2} / 2 d t
$$

is to be minimized subject to the constraints imposed by the physical system. The simplified linear system dynamics of equation 7 are assumed. The system equations are expressed in state-space form, augmented to permit explicit expression of 
jerk as a function of state and input variables $(U)$

$$
\begin{gathered}
\dot{\theta}=\omega \\
\dot{\omega}=\frac{K}{I} \theta_{0}-\frac{K}{I} \theta-\frac{B}{I} \omega \\
\dot{\theta}_{0}=U \\
\gamma=\frac{K}{I} U-\frac{K}{I} \omega-\frac{B}{I}\left\lfloor\frac{K}{I} \theta_{0}-\frac{K}{I} \theta-\frac{B}{I} \omega\right\rfloor
\end{gathered}
$$

Note that the vector of alpha motoneuron control inputs has been represented by the reference position, $\theta_{0}$.

Form the Hamiltonian:

$$
H=\frac{1}{2} \gamma^{2}+\lambda_{1} \omega+\lambda_{2}\left[\frac{K}{I} \theta_{0}-\frac{K}{I} \theta-\frac{R}{I} \omega\right]+\lambda_{3} U
$$

The Hamiltonian is to be minimized with respect to the control, $U$. If any of the assumed inequality constraints on the state or input variables become active, the active constraints must be included in the Hamiltonian (Bryson and Ho, 1975). However, if none of the inequality constraints are active, equation A6 may be brought to the extremum by partial differentiation with respect to $U$.

$$
\begin{gathered}
\frac{\partial H}{\partial U}=0 \\
U=\frac{B}{I} \theta_{0}-\frac{B}{I} \theta+\left[1-\frac{B^{2}}{K I}\right] \omega-\frac{I^{2}}{K I} \lambda_{3}
\end{gathered}
$$

This is a necessary condition, valid if none of the variables reaches its limits.

The variables $\lambda_{1}, \lambda_{2}$, and $\lambda_{3}$ are found from co-state equations:

$$
\begin{gathered}
\frac{\partial H}{\partial \theta}=-\dot{\lambda}_{1}=-\frac{K}{I} \lambda_{2}-\frac{B}{I} \lambda_{3} \\
\frac{\partial H}{\partial \omega}=-\dot{\lambda}_{2}=\lambda_{1}-\frac{B}{I} \lambda_{2}+\left[1-\frac{B^{2}}{K I}\right] \lambda_{3} \\
\frac{\partial H}{\partial \theta_{0}}=-\dot{\lambda}_{3}=\frac{K}{I} \lambda_{2}+\frac{B}{I} \lambda_{3}
\end{gathered}
$$

Combining equations A2 through A4 with equations A8 through A11 yields the following linear differential equations:

$$
\left[\begin{array}{c} 
\\
\dot{\theta} \\
\dot{\omega} \\
\dot{\theta}_{0} \\
\dot{\lambda}_{3} \\
\dot{\lambda}_{2} \\
\dot{\lambda}_{1}
\end{array}\right]=\left[\begin{array}{rrrrrr}
0 & 1 & 0 & 0 & 0 & 0 \\
-\frac{K}{I} & -\frac{B}{I} & \frac{K}{I} & 0 & 0 & 0 \\
-\frac{B}{I} & 1-\frac{B^{2}}{K I} & \frac{B}{I} & -\frac{I^{2}}{K^{2}} & 0 & 0 \\
0 & 0 & 0 & -\frac{B}{I} & -\frac{K}{I} & 0 \\
0 & 0 & 0 & -1+\frac{B^{2}}{K I} & \frac{B}{I} & -1 \\
0 & 0 & 0 & \frac{B}{I} & \frac{K}{I} & 0
\end{array}\right]\left[\begin{array}{c} 
\\
\theta \\
\omega \\
\theta_{0} \\
\lambda_{3} \\
\lambda_{2} \\
\lambda_{1}
\end{array}\right]
$$

The state equations are coupled to the co-state equations and the co-state equations are uncoupled from the state equations. The characteristic polynomial is extremely simple:

$$
s^{6}=0
$$

where $s$ is the argument of the Laplace transform.

Thus, the six eigenvalues are identically zero and the position trajectory is given by a fifth-order polynomial.

$$
\theta(t)=b_{0}+b_{1} t+b_{2} t^{2}+b_{3} t^{3}+b_{4} t^{4}+b_{5} t^{5}
$$

The reference trajectory may now be obtained by using equation A14 in A2 and A3.

\section{Appendix II}

If the function $\theta(t)$ is sufficiently differentiable in the interval $0 \leq t \leq d$ then the unconstrained functional

$$
C\{\theta(t)\}=\int_{0}^{d} F\left\{t, \theta, \dot{\theta}, \ldots, \theta^{(n)}\right\} d t
$$

assumes an extremum when $\theta(t)$ is given by the Euler-Poisson equation:

$$
\frac{\partial F}{\partial \theta}-\frac{d}{d t} \frac{\partial E}{\partial \dot{\theta}}+\cdots+(-1)^{n} \frac{d^{n}}{d t^{n}} \frac{\partial F}{\partial \theta^{(n)}}=0
$$

In this problem:

Applying equation A16:

$$
F\left\{t, \theta, \dot{\theta}, \cdots \theta^{(n)}\right\}=\gamma^{2} / 2
$$

$$
\begin{gathered}
-\frac{d^{3}}{d t^{3}}\left[\frac{\partial}{\partial \gamma}\left(\gamma^{2} / 2\right)\right]=0 \\
\therefore \frac{d^{6}}{d t^{6}} \theta=0
\end{gathered}
$$

The resulting position trajectory is given by a fifth-order polynomial:

$$
\begin{gathered}
\theta(t)=b_{0}+b_{1}+b_{2} t^{2}+b_{3} t^{3}+b_{4} t^{4}+b_{5} t^{5} \\
\text { References }
\end{gathered}
$$

Abend, W., E. Bizzi, and P. Morasso (1982) Human arm trajectory formation. Brain 105: 331-348.

Bizzi, E., and A. Polit (1979) Processes controlling visually evoked movements. Neuropsychologia 17: 203-213.

Bizzi, E., N. Accornero, W. Chapple, and N. Hogan (1981a) Processes underlying arm trajectory formation in monkeys. In Brain Mechanisms of Perceptual Awareness and Purposeful Behavior, C. AjmoneMarson and O. Pompieano, eds., pp. 311-318, Raven Press, New York.

Bizzi, E., N. Accornero, W. Chapple, and N. Hogan (1981b) Central and peripheral mechanisms in motor control. In New Perspectives in Cerebral Localization, R. A. Thompson and J. R. Green. eds., pp. 2324, Raven Press, New York.

Bizzi, E., W. Chapple, and N. Hogan (1982a) Mechanical properties of muscles: Implications for motor control. Trends Neurosci. 5: 395398.

Bizzi, E., N. Accornero, W. Chapple, and N. Hogan (1982b) Arm trajectory formation in monkeys. Exp. Brain Res. 46: 139-143.

Bizzi, E., N. Accornero, W. Chapple, and N. Hogan (1984) Posture control and trajectory formation during arm movement. J. Neurosci. 4: $2738-2744$.

Bryson, A. E., Jr., and Y. C. Ho (1975) Applied Optimal Control, John Wiley \& Sons, Inc., New York.

Buchthal, F., and H. Schmalbruch (1970) Contraction times and fiber types in intact human muscle. Acta Physiol. Scand. 79: 435-452.

Clark, M. R., and L. Stark (1975) Time-optimal behaviour of human saccadic eye movement. IEEE Trans. Automatic Control AC-20: $345-348$.

Flash, T. (1983) Organising principles underlying the formation of arm trajectories. Ph.D. dissertation, Department of Psychology, Massachusetts Institute of Technology, Cambridge, MA.

Flash, T., and N. Hogan (1982) Evidence for an optimization strategy in arm trajectory formation. Soc. Neurosci. Abstr. 8: 282.

Ghez, C., and J. H. Martin (1982) The control of rapid limb movement in the cat. III. Agonist-antagonist coupling. Exp. Brain Res. 45: 115125 .

Ghez, C., and D. Vicario (1978a) The control of rapid limb movement in the cat. I. Response latency. Exp. Brain Res. 33: 173-189.

Ghez, C., and D. Vicario (1978b) The control of rapid limb movement in the cat. II. Scaling of isometric force adjustments. Exp. Brain Res. 33: 191-202.

Hogan, N. (1979) Adaptive stiffness control in human movement. In 1979 Advances in Bioengineering, M. K. Wells, ed., pp. 53-54, American Society of Mechanical Engineers, New York.

Hogan, N. (1980a) 'Tuning muscle stiffness can simplify control of natural movement. In 1980 Advances in Bioengineering, V. C. Mow, ed., pp. 279-282. American Society of Mechanical Engineers, New York.

Hogan, N. (1980b) Mechanical impedance control in assistive devices and manipulators. In Proceedings of the 1980 Joint Automatic Controls Conference, B. Friedland and H. A. Spang, eds., Paper No. TA10-B, American Automatic Control Council, San Francisco.

Hogan, N. (1984) Adaptive control of mechanical impedance by coac tivation of antagonist muscles. IEEE Trans. Automatic Control $A C$ 29: $681-690$.

Kelso, J. A. S. (1977) Motor control mechanisms underlying human 
movement reproduction. J. Exp. Psychol. 3: 529-543.

Kelso, I. A. S., and K. G. Holt (1980) Exploring a vibratory system analysis of human movement production. J. Neurophysiol. 43: 11831196.

Lanman, J. M. (1980) Movement and the mechanical properties of the intact human elbow joint. Ph.D. dissertation, Department of Psychology, Massachusetts Institute of Technology, Cambridge, MA.

Morasso, P. (1981) Spatial control of arm movements. Exp. Brain Res. 42: $223-227$.

Nelson, W. L. (1983) Physical principles for economies of skilled movements. Biol. Cybern. 46: 135-147.

Polit, A., and E. Bizzi (1978) Processes controlling arm movements in monkeys. Science 102: 1235-1237.

Polit, A., and E. Bizzi (1979) Characteristics of motor programs underlying arm movements in monkeys. J. Neurophysiol. 42: 183-194.

Pontryagin, I. S., V. Boltyanskii, R. Gamkrelidze, and E. Mishchenko
(1962) The Mathematical Theory of Optimal Processes, Interscience Publishers, Inc., New York.

Schmidt, R. A., and C. McGown (1980) Terminal accuracy of unexpectedly loaded rapid movements: Evidence for a mass-spring mechanism in programming. J. Motor Behav. 12: 149-161.

Stein, R. B. (1982) What muscle variable(s) does the nervous system control in limb movements? Behav. Brain Sci. 5: 535-577.

Viviani, P., and C. Terzuolo (1980) Space-time invariance in motor skills. In Tutorials in Motor Behaviour, G. E. Stelmach and J. Requin, eds., pp. 525-533, Elsevier-North-Holland Publishing Co., Amsterdam.

Whiting, H. T. A. (1984) Human Motor Actions: Bernstein Reassessed, Elsevier Science Publishing Company, Amsterdam.

Zahalak, G. I., and S. J. Heyman (1979) A quantitative evaluation of the frequency response characteristics of active human skeletal muscle in vivo. J. Biomech. Eng. 101: 28-37. 